\title{
Integration of Highly Oscillatory Problems Through $G$-Functions
}

\author{
F. García-Alonso, J.A. Reyes, and Y. Villacampa*
}

Departamento de Matemática Aplicada, Escuela Politécnica Superior, Universidad de Alicante, Spain

\begin{abstract}
Perturbed harmonic oscillators describe many models in physics and engineering. A method for solving this type of problem is based on the utilization of Scheifele's functions, consisting of a refinement of the Taylor series method. One disadvantage of the method is that it is difficult to determine, in each case, the recurrence relations necessary for arriving to the solutions. In this paper we construct the numerical method especially adapted to the integration of oscillators, using developments in the form of $G$-functions. In addition, two computer applications related to highly oscillatory problems are implemented, and recurrence relations are determined in each one. The results show better precision in the application of $G$-functions, compared to other known methods implemented in MAPLE V.
\end{abstract}

Keywords: Numerical solutions of ODE's, Highly accurate solutions, Highly oscillatory problems.

\section{INTRODUCTION}

In nature, there are cases of bodies that move through the same positions repeatedly, in equal time intervals and always with the same velocity and acceleration. This is called periodic motion. As we know, simple harmonic vibratory motion is described by equations $x(t)=A \cos \left(\omega t+\varphi_{0}\right)$ or $x(t)=A \sin \left(\omega t+\varphi_{0}\right)$, the solution of a linear differential equation of the second order, $\ddot{x}(t)+\omega^{2} x(t)=0$, homogeneous and constant coefficients, where the points indicate derivation with regard to time, following Newton's notation. If a frictional force is considered, we obtain $\ddot{x}(t)+2 \gamma \dot{x}(t)+\omega^{2} x(t)=0$, and if an exterior force $F(t)$, is also included, the damped and driven - i.e., perturbed - harmonic oscillator allows for the expression $\ddot{x}(t)+2 \gamma \dot{x}(t)+\omega^{2} x(t)=F(t)$. There are numerous processes in physics that can be adapted to a model by means of perturbed oscillators.

In 1971, Scheifele [1] devised a method for the numerical integration of perturbed oscillators based on a refinement of the classic Taylor series method, using a sequence of functions $G_{k}(t)$ permitting expression of the solution in terms of a series of the type $x(t)=\sum_{k=0}^{\infty} b_{k} G_{k}(t)$, used in the construction of a very precise numerical integration method. This method not only has the advantage of integrating exactly a non-perturbative problem, but also of integrating it with only the first two terms of the series, while the Taylor series method provides an approximation of the solution through a linear function, if we take the first two terms.

In addition to constructing the Scheifele $G$-functions and revealing their most relevant properties, this paper present on the numerical integration method based on developments in the form of $G$-functions for perturbed oscillators, ending with a detailed study of two perturbed oscillators, illustrating how they are employed and contrasting their behaviour. For this propose their degrees of precision are compared with

*Address correspondence to this author at the Departamento de Matemática Aplicada, Escuela Politécnica Superior, Universidad de Alicante, Spain; E-mail: villacampa@ua.es those of well-known codes such as RKF45, LSODE and GEAR [2,3] implemented in MAPLE V, with the goal ofguaranteeing that the results are not distorted by poor programming that favours the method based on $G$-functions.

\section{SCHEIFELE'S $G$-FUNCTIONS}

Let $x(t)$ be the solution of the perturbed oscillator in equation:

$$
\begin{aligned}
& x^{\prime \prime}+\alpha x=\varepsilon \cdot f\left(x, x^{\prime}, t\right), \\
& x(0)=x_{0}, \\
& x^{\prime}(0)=x_{0}^{\prime},
\end{aligned}
$$

assuming that the function $g(t)=f\left(x(t), x^{\prime}(t), t\right)$ admits an expansion in the form:

$$
g(t)=\sum_{n=0}^{\infty} c_{n} \frac{t^{n}}{n !} \text { con } c_{n}=g^{n)}(0),
$$

with which (1) can be written:

$$
\begin{aligned}
& x^{\prime \prime}+\alpha x=\varepsilon \cdot \sum_{n=0}^{\infty} c_{n} \frac{t^{n}}{n !}, \\
& x(0)=x_{0}, \\
& x^{\prime}(0)=x_{0}^{\prime} .
\end{aligned}
$$

The solution to the above problem can be obtained in the habitual manner, calculating the solution of the homogeneous equation with the initial conditions and adding on the particular solution of the non-homogeneous equation, in which the solution and its derivative is annulled by setting $t=0$. The latter can be calculated by applying the principle of superposition of solution on IVP's:

$$
\begin{aligned}
& x_{n}^{\prime \prime}+\alpha x_{n}=\frac{t^{n}}{n !}, \\
& x_{n}(0)=x_{n}^{\prime}(0)=0, \forall n \geq 0
\end{aligned}
$$

combining the solutions in linear form with the coefficients of $\varepsilon \cdot c_{n}$. 
Based on this idea, G. Scheifele [1] defined a collection of special functions adapted to the solution of this type of problem.

Scheifele's $G$-functions verify:

$G_{n}(t)=x_{n-2}(t), \quad \forall n \geq 2$,

where $x_{n}(t)$ are solutions of (4); in other words, the $G_{n}$ functions satisfy $\forall n \geq 2$ :

$G_{n}^{\prime \prime}(t)+\alpha G_{n}(t)=\frac{t^{n-2}}{(n-2) !}$,

$G_{n}(0)=G_{n}^{\prime}(0)=0$.

these functions, defined in this way, verify that:

$G_{n}^{\prime}(t)=G_{n-1}(t), \quad \forall n \geq 3$.

Thus, all the $G$-functions can be calculated from $G_{2}$, but Scheifele's first two and most important functions, $G_{0}^{2}$ and $G_{1}$, are introduced as solutions of homogeneous problems:

$x^{\prime \prime}+\alpha x=0$,

$x(0)=1$,

$x^{\prime}(0)=0$.

$x^{\prime \prime}+\alpha x=0$,

$x(0)=0$,

$x^{\prime}(0)=1$.

Functions $G_{0}(t)$ and $G_{1}(t)$ also verify the property:

$G_{2}^{\prime}(t)=G_{1}(t), G_{1}^{\prime}(t)=G_{0}(t)$.

Combining (7) and (10), we can affirm that:

$G_{n}^{\prime}(t)=G_{n-1}(t), n \geq 1$.

Taking into account (6) and (11), we obtain the following recurrence relation:

$G_{n}(t)+\alpha G_{n+2}(t)=\frac{t^{n}}{n !}, \quad \forall n \geq 0$.

This recurrence relation will be very useful in the calculation of $G$-functions, and therein lies its importance. Another relevant property in the calculation of $G$-functions is that they can be expressed through absolutely convergent series for every value of $t$ :

$G_{n}(t)=\sum_{n=0}^{\infty} \beta_{j} \frac{t^{j+n}}{(j+n) !}, \quad n \geq 0$,

where $\beta_{2 j}=(-\alpha)^{j}$ and $\beta_{2 j+1}=0, \forall j \geq 0$.

Its importance lies in the fact that, by truncating the series, it provides an alternative method for calculating $G$ functions. is:

The solution to the problem (3) in terms of G-functions

$$
x(t)=x_{0} G_{0}(t)+x_{0}^{\prime} G_{1}(t)+\varepsilon \sum_{n=0}^{\infty} c_{n} G_{n+2}(t) .
$$

This result will allow us to define the numerical integration method based on $G$-functions.

\section{FINITE TAYLOR SERIES AND $G$-EXPANSIONS}

Building the Taylor expansion and truncating the solution $x(t)$ of (1), we obtain an approximation of the solution:

$x_{m}(t)=\sum_{k=0}^{m} \frac{t^{k}}{k !} a_{k}$ with $a_{k}=x^{k)}(0)$.

By replacing relation (12) in (15), we obtain [1]:

$$
\begin{aligned}
& x_{m}(t)=G_{0}(t) a_{0}+G_{1}(t) a_{1}+\sum_{k=2}^{m} G_{k}(t)\left(a_{k}+\alpha a_{k-2}\right)+ \\
& +\alpha G_{m+1}(t) a_{m-1}+\alpha G_{m+2}(t) a_{m} .
\end{aligned}
$$

Defining a new sequence of coefficients $b_{k}$, in the following manner:

$$
\begin{aligned}
& b_{0}=a_{0}, \\
& b_{1}=a_{1}, \\
& b_{k}=a_{k}+\alpha a_{k-2} \forall k \geq 2
\end{aligned}
$$

(16) is reduced to:

$$
x_{m}(t)=\sum_{k=0}^{m} G_{k}(t) b_{k}+\alpha\left(G_{m+1}(t) a_{m-1}+G_{m+2}(t) a_{m}\right),
$$

and eliminating the last term results in a different approximation,

$$
X_{m}(t)=\sum_{k=0}^{m} G_{k}(t) b_{k},
$$

that can be considered the extension of the Taylor series for the solution of (3), which provides greater precision than $x_{m}(t)$.

The coefficients of

$x_{m}(t)=\sum_{k=0}^{m} \frac{t^{k}}{k !} a_{k}$ and $X_{m}(t)=\sum_{k=0}^{m} G_{k}(t) b_{k}$ for (3) are provided by:

$$
\begin{aligned}
& a_{0}=x_{0}, \\
& a_{1}=x_{0}^{\prime}, \\
& a_{k+2}+\alpha a_{k}=\varepsilon g^{k)}(0) k \geq 0 \\
& b_{0}=x_{0}, \\
& b_{1}=x_{0}^{\prime}, \\
& b_{k+2}=\varepsilon g^{k)}(0) k \geq 0,
\end{aligned}
$$

therefore:

$$
\begin{aligned}
& X_{m}(t)=\sum_{k=0}^{m} G_{k}(t) b_{k}= \\
& =G_{0}(t) x_{0}+G_{1}(t) x_{0}^{\prime}+\varepsilon \sum_{k=2}^{m} g^{k-2)}(0) G_{k}(t) .
\end{aligned}
$$

Inserting this last result in (3), the residual $R_{m}(t)$, corresponding to $X_{m}(t)$, is obtained:

$$
R_{m}(t)=\varepsilon g(t)-\left(X_{m}^{\prime \prime}(t)+\alpha X_{m}(t)\right)=\varepsilon \sum_{k=0}^{\infty} \frac{t^{k}}{k !} g^{k)}(0) .
$$


Taking into account:

$$
\begin{aligned}
& x_{m}^{\prime \prime}(t)+\alpha x_{m}(t)=\sum_{k=2}^{m}\left(G_{k-2}(t)+\alpha G_{k}(t)\right) b_{k}+ \\
& +\alpha\left(a_{m-1}\left(G_{m-1}(t)+\alpha G_{m+1}(t)\right)+a_{m}\left(G_{m}(t)+\alpha G_{m+2}(t)\right)\right)
\end{aligned}
$$

and (12), the residual $r_{m}(t)$ corresponding to $x_{m}(t)$ is:

$r_{m}(t)=\varepsilon g(t)-\left(x_{m}^{\prime \prime}(t)+\alpha x_{m}(t)\right)=$

$=\varepsilon \sum_{k=m-1}^{\infty} \frac{t^{k}}{k !} g^{k)}(0)-\alpha\left(a_{m-1} \frac{t^{m-1}}{(m-1) !}+a_{m} \frac{t^{m}}{m !}\right)$.

From all the above, it can be concluded that while in $R_{m}(t)$ the parameter of perturbation $\varepsilon$ is a factor, in $r_{m}(t)$ it is not. As a result, $R_{m}(t)$ will be small with $\varepsilon$, but $r_{m}(t)$ will not. If $\varepsilon=0$, the power series method results in error, while the Scheifele $G$-function method, with only the first and second term, integrates the equation exactly.

\section{THE $G$-FUNCTIONS SERIES METHOD}

For the construction of a numerical method based on the $G$-functions in (1), let us assume that both the solution $x(t)$ and its perturbation function $g(t)=f\left(x(t), x^{\prime}(t), t\right)$ are analytical functions.

Once all the $G$-functions necessary for truncation of the series that define them are calculated, to obtain an approximation of the solution $x(t)$ we replace a truncation of its solution in (3), which allows us to establish recurrence relations for calculating the coefficients $c_{k}=g^{k)}(0)$, starting with $x_{0}$ and $x_{0}^{\prime}$. Once the coefficients $c_{k}$ are calculated for $k=0, \ldots, m-2$ and step $h$ is set, the approximation of the solution and its derivative in point $h$ result respectively in the expressions:

$$
\begin{aligned}
& x_{1}=G_{0}(h) x_{0}+G_{1}(h) x_{0}^{\prime}+\varepsilon \sum_{k=0}^{m-2} c_{k} G_{k+2}(h) \\
& x_{1}^{\prime}=G_{0}(h) x_{0}^{\prime}-\alpha G_{1}(h) x_{0}+\varepsilon \sum_{k=0}^{m-2} c_{k} G_{k+1}(h) .
\end{aligned}
$$

Clearly, these approximations verify:

$x^{\prime \prime}+\alpha x=\varepsilon \cdot f\left(x, x^{\prime}, t\right)$,

$x(h)=x_{1}$,

$x^{\prime}(h)=x_{1}^{\prime}$.

Let's suppose that we have already calculated an approximation of the solution and its derivative in point $t=n h, x_{n}$ and $x_{n}^{\prime}$, verifying:

$x^{\prime \prime}+\alpha x=\varepsilon \cdot f\left(x, x^{\prime}, t\right)$,

$x(n h)=x_{n}$,

$x^{\prime}(n h)=x_{n}^{\prime}$.

To calculate an approximation of the solution and its derivative in point $(n+1) h$, it is convenient to make a change of time origin $t=\tau+n h$, yielding:

$x^{\prime \prime}+\alpha x=\varepsilon \cdot f\left(x, x^{\prime}, \tau+n h\right)$,

$x(0)=x_{n}$, $x^{\prime}(0)=x_{n}^{\prime}$

bringing us to the initial situation. We calculate the coefficients by recurrence:

$f\left(x(\tau), x^{\prime}(\tau), \tau+n h\right)=\sum_{k=0}^{\infty} \frac{\tau^{k}}{k !} c_{k}$, with

$c_{k}=\frac{d^{k} g(0)}{d \tau^{k}}=\frac{d^{k} g(n h)}{d t^{k}}$

and the approximation of the solution and its derivative in point $(n+1) h$ resulting in the formulas:

$$
\begin{aligned}
& x_{n+1}=G_{0}(h) x_{n}+G_{1}(h) x_{n}^{\prime}+\varepsilon \sum_{k=0}^{m-2} c_{k} G_{k+2}(h), \\
& x_{n+1}^{\prime}=G_{0}(h) x_{n}^{\prime}-\alpha G_{1}(h) x_{n}+\varepsilon \sum_{k=0}^{m-2} c_{k} G_{k+1}(h),
\end{aligned}
$$

respectively, which constitute the numerical integration method for perturbative harmonic oscillators based on Scheifele's $G$-functions.

\section{NUMERICAL EXPERIMENTS}

In this section, we have selected two examples that provide a good look at this integrator's behaviour, and the recurrence relations are determined in each one.

\section{Example I}

Let's consider the highly oscillatory problem proposed by Petzold [4], which contains a harmonic oscillator with a perturbation function explicitly dependent on time:

$x^{\prime \prime}+\alpha^{2} x=A \sin (\alpha t)$,

with $x(0)=1, x^{\prime}(0)=-\frac{A}{2 \alpha}$ and $t \in[0,10]$,

which the exact solution and its derivative are expressed in the equations:

$$
\begin{aligned}
& x(t)=\left(1-\frac{A t}{2 \alpha}\right) \cos (\alpha t), \\
& x^{\prime}(t)=\frac{A t-2 \alpha}{2} \sin (\alpha t)-\frac{A}{2 \alpha} \cos (\alpha t) .
\end{aligned}
$$

Although its solution can be calculated exactly by means of analytical procedures, this example has been chosen to illustrate how well the $G$-functions method works for highly oscillatory harmonic perturbation functions. tained:

In the first integration step, the recurrence relation is ob-

$a_{0}=x_{0}$

$a_{1}=x_{0}^{\prime}$,

$a_{k+2}=-\alpha^{2} a_{k}+\varepsilon \alpha^{k} \sin \left(\frac{k \pi}{2}\right) \forall k \geq 0$

and coefficients $b_{k}$ are calculated through the relations:

$b_{0}=a_{0}$,

$b_{1}=a_{1}$, 
$b_{k+2}=a_{k+2}+\alpha^{2} a_{k}$.

Noting that the approximations of $x(h)$ and $x^{\prime}(h)$ are $x_{1}$ and $x_{1}^{\prime}$, respectively, the approximation of the solution and its derivative result in:

$$
\begin{aligned}
& x_{1}=G_{0}(h) x_{0}+G_{1}(h) x_{0}^{\prime}+\varepsilon \sum_{k=0}^{m-2} c_{k} G_{k+2}(h), \\
& x_{1}^{\prime}=G_{0}(h) x_{0}^{\prime}-\alpha G_{1}(h) x_{0}+\varepsilon \sum_{k=0}^{m-2} c_{k} G_{k+1}(h)
\end{aligned}
$$

In order to carry out the second step of integration, it is necessary to solve the following IVP:

$x^{\prime \prime}+\alpha^{2} x=\varepsilon \sin (\alpha t)$,

$x(h)=x_{1}$,

$x^{\prime}(h)=x_{1}^{\prime}$,

which has the disadvantage that, when setting a step size $h$ and calculating the value of the approximation $x(h)$ and $x^{\prime}(h)$, it is not possible to move to a second step, since the perturbation function depends explicitly on time. To avoid this difficulty, we proceed in the following manner:

The independent variable $\tau=t-h$ is changed, and $x_{*}(t)=\left(x^{*} \circ \tau\right)(t)$ is considered. By replacing $x(t)$ with $x(\tau)$ in (37), we obtain:

$x^{* \prime \prime}+\alpha x^{*}=\varepsilon \sin (\alpha(\tau+h))$,

$x^{*}(0)=x(h)=x_{1}$,

$x^{* \prime}(0)=x^{\prime}(h)=x_{1}^{\prime}$.

This strategy makes it possible to initialize the method and obtain the recurrences:

$a_{0}=x^{*}(0)=x_{1}$,

$a_{1}=x^{* \prime}(0)=x_{1}^{\prime}$,

$a_{k+2}+\alpha^{2} a_{k}=\varepsilon \alpha^{k} \sin \left(\alpha h+\frac{k \pi}{2}\right) \forall k \geq 0$,

$b_{0}=a_{0}$,

$b_{1}=a_{1}$,

$b_{k+2}=a_{k+2}+\alpha^{2} a_{k}$.

The approximation of the solution $x(t)$ and $x^{\prime}(t)$ in $t=2 h$, result in the formulas:

$x_{2}=G_{0}(h) x_{1}+G_{1}(h) x_{1}^{\prime}+\varepsilon \sum_{k=0}^{m-2} c_{k} G_{k+2}(h)$,

$x_{2}^{\prime}=G_{0}(h) x_{1}^{\prime}-\alpha G_{1}(h) x_{1}+\varepsilon \sum_{k=0}^{m-2} c_{k} G_{k+1}(h)$.

For a third integration step, it is necessary to solve the following IVP:

$x^{\prime \prime}+\alpha^{2} x=\varepsilon \sin (\alpha t)$,

$x(2 h)=x_{2}$,

$x^{\prime}(2 h)=x_{2}^{\prime}$.
The independent variable $\tau=t-2 h$ is changed, and is considered. $x(t)=(x \circ \tau)(t)$. By replacing $x(t)$ with $x^{*}(\tau)$ in (42), we obtain:

$x^{* \prime \prime}+\alpha x^{*}=\varepsilon \sin (100(\tau+2 h))$,

$x^{*}(0)=x(2 h)=x_{2}$,

$x^{* \prime}(0)=x^{\prime}(2 h)=x_{2}^{\prime}$.

In this way, the method is initialized again and we obtain the recurrences:

$a_{0}=x^{*}(0)=x_{2}$,

$a_{1}=x^{* \prime}(0)=x_{2}^{\prime}$,

$a_{k+2}+\alpha^{2} a_{k}=\varepsilon \alpha^{k} \sin \left(2 \alpha h+\frac{k \pi}{2}\right) \forall k \geq 0$,

$b_{0}=a_{0}$,

$b_{1}=a_{1}$,

$b_{k+2}=a_{k+2}+\alpha^{2} a_{k}$.

The approximation of the solution $x(t)$ and $x^{\prime}(t)$ in $t=3 h$, result in the formulas:

$x_{3}=G_{0}(h) x_{2}+G_{1}(h) x_{2}^{\prime}+\varepsilon \sum_{k=0}^{m-2} c_{k} G_{k+2}(h)$,

$x_{3}^{\prime}=G_{0}(h) x_{2}^{\prime}-\alpha G_{1}(h) x_{2}+\varepsilon \sum_{k=0}^{m-2} c_{k} G_{k+1}(h)$.

This procedure resolves the difficulty that the perturbation function depends explicitly on time, and allows us to maintain, throughout the process of integration, the same $G$ functions calculated for the initial conditions given in the origin.

Fig. (1) contrasts the graph of the absolute value decimal logarithm of the relative error of the solution $x(t)$, calculated using Scheifele's method with $17 G$-functions, $A=1$, step size $h=0.01$ and $\alpha=10$, with the numerical integration codes LSODE, RKF45 and GEAR, all with a tolerance of $10^{-}$ 15 .

Fig. (2), contrasts its derivative $x^{\prime}(t)$ under the same conditions:

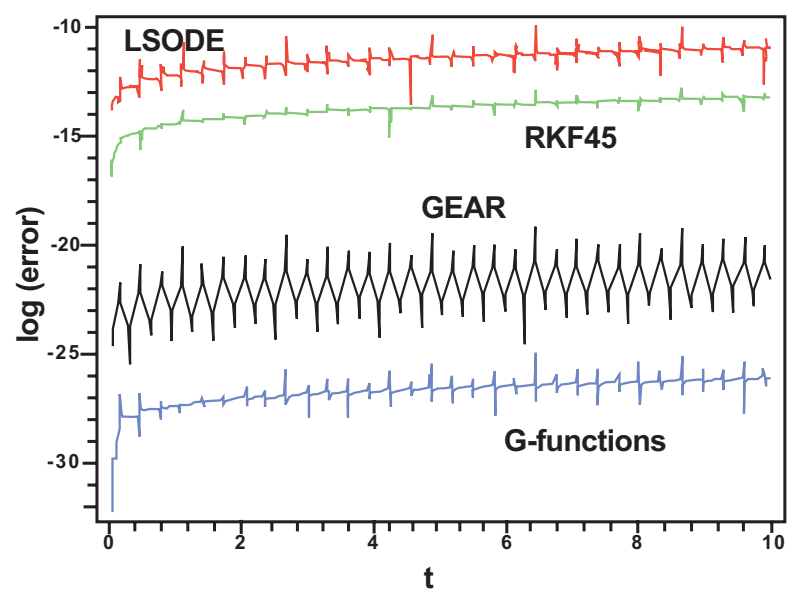

Fig. (1). $x^{\prime \prime}+100 x=\varepsilon \sin (10 t)$ with $\mathrm{h}=0.01$ (position $\mathrm{x}(\mathrm{t})$ ). 


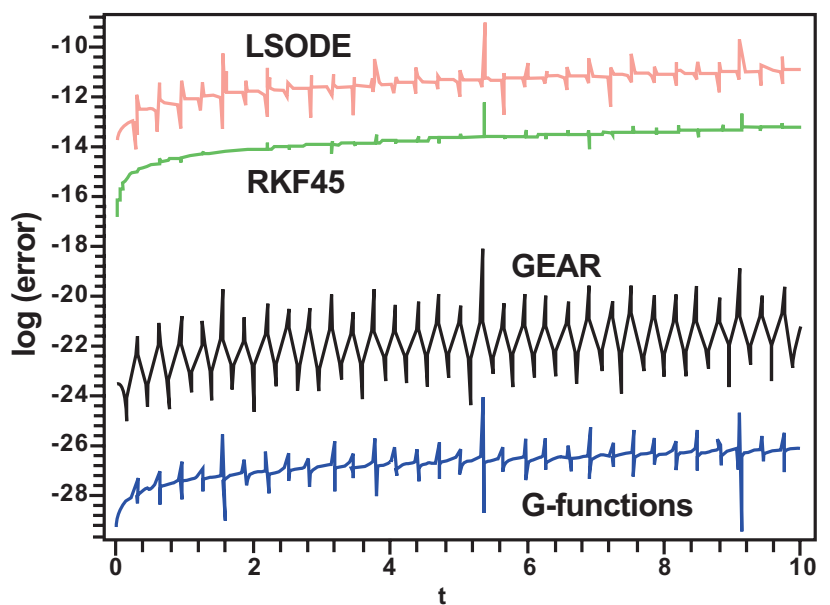

Fig. (2). $x^{\prime \prime}+100 x=\varepsilon \sin (10 t)$ with $\mathrm{h}=0.01$ (velocity $\mathrm{x}^{\prime}(\mathrm{t})$ ).

\section{Example II}

G. Denk [5] proposed the following highly oscillatory problem, to contrast a new multiple-step method he designed for the integration of highly oscillatory problems.

$x^{\prime \prime}+\kappa^{2} x=\kappa^{2} t$

$x(0)=10^{-5}$,

$x^{\prime}(0)=1-\kappa 10^{-5} \frac{\cos \kappa}{\sin \kappa}$,

$\kappa=314.16$,

with the exact solution:

$x(t)=t+10^{-5}\left(\cos (\kappa t)-\frac{\cos \kappa}{\sin \kappa} \sin (\kappa t)\right)$,

$x^{\prime}(t)=1-10^{-5} \kappa\left(\sin (\kappa t)+\frac{\cos \kappa}{\sin \kappa} \cos (\kappa t)\right)$.

As in the previous example, by replacing the appropriate series in (47) we obtain the recurrences:

$a_{0}=x_{0}$,

$a_{1}=x_{0}^{\prime}$,

$a_{2}=-\kappa^{2} a_{0}$

$a_{3}=-\kappa^{2} a_{1}+\kappa^{2}$,

$a_{k+2}=-\kappa^{2} a_{k} \quad \forall k \geq 2$

and the coefficients $b_{k}$ in $G$-functions are calculated by means of:

$b_{0}=a_{0}=x_{0}$,

$b_{1}=a_{1}=x_{0}^{\prime}$,

$b_{k+2}=a_{k+2}+\kappa^{2} a_{k} \forall k \geq 0$

The approximation of the solution $x(t)$ and $x^{\prime}(t)$ in $t=h$, result in the formulas:

$$
\begin{aligned}
& x_{1}=G_{0}(h) x_{0}+G_{1}(h) x_{0}^{\prime}+\varepsilon \sum_{k=0}^{m-2} c_{k} G_{k+2}(h), \\
& x_{1}^{\prime}=G_{0}(h) x_{0}^{\prime}-\alpha G_{1}(h) x_{0}+\varepsilon \sum_{k=0}^{m-2} c_{k} G_{k+1}(h) .
\end{aligned}
$$

It is not possible to calculate a second integration step directly, because the perturbation function depends explicitly on $t$, which requires solving the IVP:

$x^{\prime \prime}+\kappa^{2} x=\kappa^{2} t$,

$x(h)=x_{1}$,

$x^{\prime}(h)=x_{1}^{\prime}$,

$\kappa=314.16$,

Proceeding as in the previous example, the independent variable $\tau=t-h$ is changed and $x(t)=\left(x^{*} \circ \tau\right)(t)$ is considered. By replacing $x(t)$ with $x(\tau)$ in $(52)$, we obtain:

$x^{* \prime \prime}+\kappa^{2} x^{*}=\kappa^{2}(\tau+h)$,

$x^{*}(0)=x(h)=x_{1}$,

$x^{* \prime}(0)=x^{\prime}(h)=x_{1}^{\prime}$,

This strategy allows us to reinitiate the method and obtain the recurrences:

$$
\begin{aligned}
& a_{0}=x^{*}(0)=x_{1}, \\
& a_{1}=x^{* \prime}(0)=x_{1}^{\prime}, \\
& a_{2}=-\kappa^{2} a_{0}+\kappa^{2} h, \\
& a_{3}=-\kappa^{2} a_{1}+\kappa^{2}, \\
& a_{k+2}+\kappa^{2} a_{k}=0 \quad \forall k \geq 2, \\
& b_{0}=a_{0}, \\
& b_{1}=a_{1}, \\
& b_{k+2}=a_{k+2}+\kappa^{2} a_{k} \quad \forall k \geq 0 . \\
& x_{2}=G_{0}(h) x_{1}+G_{1}(h) x_{1}^{\prime}+\varepsilon \sum_{k=0}^{m-2} c_{k} G_{k+2}(h), \\
& x_{2}^{\prime}=G_{0}(h) x_{1}^{\prime}-\alpha G_{1}(h) x_{1}+\varepsilon \sum_{k=0}^{m-2} c_{k} G_{k+1}(h) .
\end{aligned}
$$

This resolves the problem of the perturbation function's explicit dependence on time.

Fig. (3) contrasts the graph of the absolute value decimal logarithm of the relative error of the solution $x(t)$, calculated using Scheifele's method with $5 G$-functions, step size $h=0.01$, with the numerical integration codes LSODE, RKF45 and GEAR, all with a tolerance of $10^{-15}$. tions.

Fig. (4) contrasts derivative $x^{\prime}(t)$, under the same condi- 


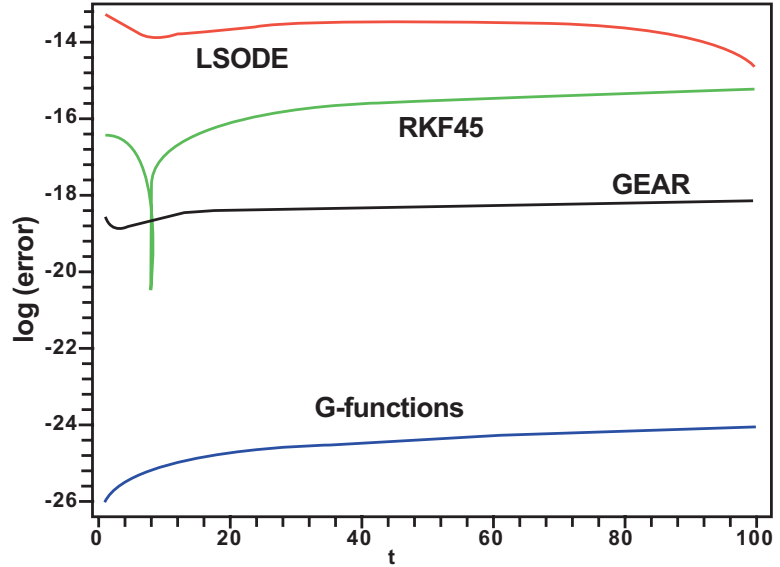

Fig. (3). $x^{\prime \prime}+\kappa^{2} x=\kappa^{2} t$ with $\mathrm{h}=0.1($ position $\mathrm{x}(\mathrm{t}))$.

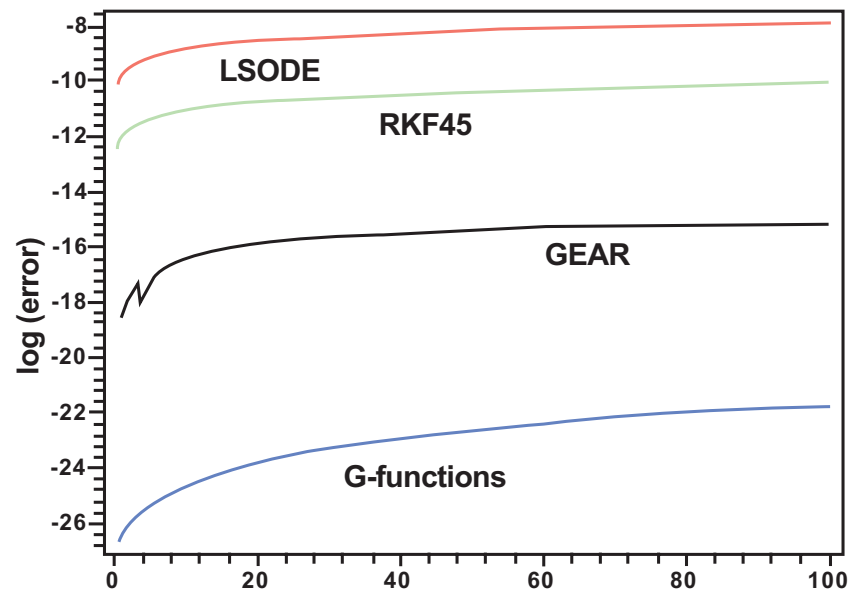

Fig. (4). $x^{\prime \prime}+\kappa^{2} x=\kappa^{2} t$ con $\mathrm{h}=0.1\left(\right.$ velocity $\left.\mathrm{x}^{\prime}(\mathrm{t})\right)$.

\section{CONCLUSIONS}

The theoretical construction of Scheifele's functions allows us to formulate solutions in $G$-functions as an exten- sion of the well-known Taylor series, with the advantage not only of integrating unperturbed problems exactly, but also of integrating them with only the first two terms of the series. As the numerical experiments show, the $G$-function method's greater precision makes it a useful tool.

The $G$-function method can be generalized with relative ease to the integration of linear differential equation systems with constant coefficients, maintaining the good properties for equations. The ideas expressed in this numerical method lay the foundations for other, more advanced multiple-step codes, also based on $G$-functions or on a refinement of them for systems, such as the SMF [6,7], SVF [8] and $\mathrm{EI}_{p} \mathrm{PC}$ [9] methods.

\section{REFERENCES}

[1] Stiefel EL, Scheifele G. Linear and regular celestial mechanics, Springer, New York 1971.

[2] Gear CW. Numerical initial value problems in ordinary differential equations, Prentice-Hall 1971.

[3] Ola Fatunla S. Numerical methods for initial value problems in ordinary differential equations, Academic Press Inc., New York 1988.

[4] Palacios M. Métodos multi-revolución simétricos para propagación de órbitas en intervalos grandes de tiempo. Monogr Real Acad Ciencias Zaragoza, 2003; 22: 55-66.

[5] Denk G. A new numerical method for the integration of highly oscillatory second-order ordinary differential equations. Appl Num Math 1993; 13: 57-67.

[6] Martín P, Ferrándiz JM. Behaviour of the SMF method for the numerical integration of satellite orbits. Celestial Mechanics Dynamic Astron 1995; 63: 29-40.

[7] Martín P, and Ferrándiz JM. Multistep numerical methods based on Scheifele G-functions with application to satellite dynamics, SIAM. J Numer Anal 1997; 34: 359-375.

[8] Vigo-Aguiar JJ, and Ferrándiz JM. Higher-order variable-step algorithms adapted to the accurate numerical integration of perturbed oscillators. Comput Phys 1998; 12(5): 467-470.

[9] Reyes JA, García-Alonso F, Ferrándiz JM, Vigo-Aguiar J. Numeric multistep variable methods for perturbed linear system integration. Appl Math Comput 2007; 190: 63-79. 